\title{
СРАВНЕНИЕ РЕЖИМОВ СКАНИРОВАНИЯ КОНУСНО-ЛУЧЕВОЙ КОМПЬЮТЕРНОЙ ТОМОГРАФИИ ДЛЯ ОЦЕНКИ РАЗМЕРОВ ДЕФЕКТОВ НАРУЖНОЙ КОРТИКАЛЬНОЙ ПЛАСТИНКИ У ПАЦИЕНТОВ С РЕЦЕССИЕЙ ДЕСНЫ В ОБЛАСТИ ФРОНТАЛЬНОЙ ГРУППЫ ЗУБОВ НИЖНЕЙ ЧЕЛЮСТИ
}

\section{COMPARISON OF TWO MODES \\ OF CONE-BEAN COMPUTED \\ TOMOGRAPHY FOR THE ASSESSMENT DEFECTS OF BUCCAL BONE PLATE IN ANTERIOR MANDIBULAE}

\section{N. Bovanova}

G. Golubeva

Summary. The aim of this study was to evaluate the effect of voxel size in two different modes of CT scanning on the visualisation of the morphology of buccal cortical plate. Twenty CT scans of patients who underwent gingival plastic surgery in the mandibular anterior region were studied. CBCT data were obtained in two modes with a voxel size of $0.16 \mathrm{~mm} 3$ and $0.4 \mathrm{~mm} 3$. The surgical data and CT data were compared by Pirson correlation coefficient.

Voxel size $0.16 \mathrm{~mm} 3$ can be recommended for the assessment of the morphology of the buccal cortical plate. However, it is possible to overestimate the actual size of intrabony defects.

Keywords: cone beam computed tomography, gingival recession, buccal cortical plate, voxel size.

\author{
Бованова Надежда Викторовна \\ Аспирант, ФГАОУ ВО «Первый Московский \\ государственный медицинский университет имени \\ И.М. Сеченова» Минздрава России (Сеченовский \\ университет) \\ nadyab92@mail.ru \\ Голубева Галина Иванова \\ Врач-рентгенолог, ООО «3 Д» независимые \\ рентгендиагностические лаборатории
}

Аннотация. Цель данного исследования — оценить влияние размера воксельного элемента в двух режимах сканирования КЛКТ на визуализацию морфологии наружной кортикальной пластинки. Было изучено 20 компьютерных томограмм пациентов, которым были проведены операции по ликвидации рецессии десны в области фронтального отдела нижней челюсти. Данные КЛКТ получены на в режимах с размером вокселя $0,16 \mathrm{~mm}^{3}$ и 0,4 мм³. Проведено сравнение данных, полученных в ходе операционного вмешательства, и данных КЛКТ с помощью метода корреляции Пирсона.

Метод КЛКТ с использование режима сканирования с размером воксельного элемента 0,16 мм³ может быть рекомендован для оценки архитектоники наружной кортикальной пластинки. Однако, возможна переоценка фактических размеров внутрикостных дефектов.

Ключевые слова: конусно-лучевая компьютерная томография, рецессия десны, наружная кортикальная пластинка, размер вокселя.

техническими характеристиками, но наиболее важным параметром, влияющим на качество получаемых данных, является размер воксельного элемента [4,7,11]. Размер вокселя влияет на разрешение и качество изображения, а также на время сканирования и реконструкцию изображения $[1,4,10,11]$.

Ранее были проведены исследования возможности метода КЛКТ оценить морфологию наружной кортикальной пластинки челюстей in vivo. В частности, Sun et al (2015), Timock et al (2011) исследовали точность визуализации дегисценций (щелевидный дефект) и фенестраций (окончатый дефект) в области фронтального отдела верхней челюсти $[12,13]$. Однако, ранее не были проведены исследования возможности метода КЛКТ для 
Таблица 1. Технические характеристики режимов сканирования.

\begin{tabular}{|l|l|l|}
\hline Характеристика & $\begin{array}{l}\text { 3D Accuitomo 170 (J. Morita Mfg., Corp., } \\
\text { Kyoto, Japan) }\end{array}$ & $\begin{array}{l}\text { Planmeca ProMax 3D (Planmeca OY, } \\
\text { Helsinki, Finland) }\end{array}$ \\
\hline Размер FOV, cm & $8^{*} 8$ & $27,5^{*} 27,5$ \\
\hline Изотропный размер вокселя, mm3 & 0,16 & 0,4 \\
\hline Напряжение, kV & 90 & 120 \\
\hline Сила тока, mA & 8 & 6 \\
\hline Время сканирования, сек & 30,8 & 20 \\
\hline Угол вращения датчика & 360 & 270 \\
\hline Зона исследования & $\begin{array}{l}\text { Верхняя и нижняя челюсти, дно } \\
\text { верхнечелюстных пазух }\end{array}$ \\
\hline Показание для исследования & $\begin{array}{l}\text { Планирование терапевтического } \\
\text { и имплантологического лечения }\end{array}$ & Череп \\
\hline
\end{tabular}

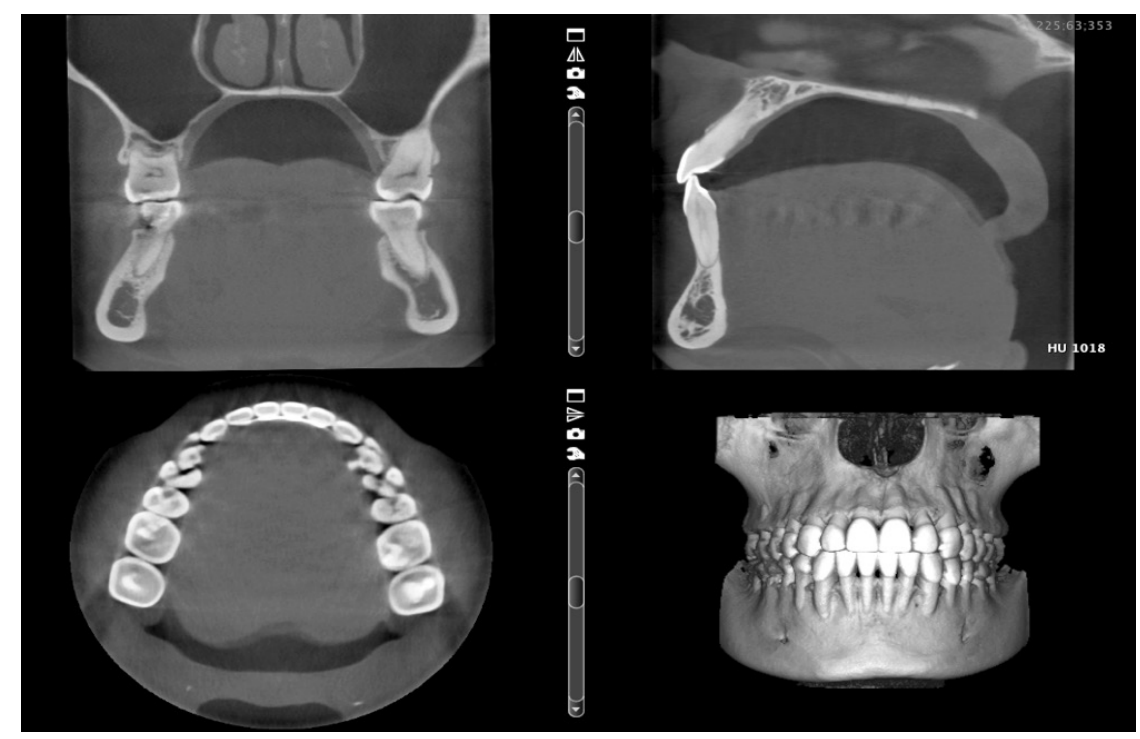

Рис. 1. Пример данных КЛКТ, полученных с помощью томографа 3D Accuitomo 170 (J. Morita Mfg., Corp., Kyoto, Japan)

визуализации наружной кортикальной пластинки фронтального отдела нижней челюсти.

Целью данного исследования оценить влияние размера воксельного элемента в двух режимах сканирования КЛКТ на визуализацию морфологии наружной кортикальной пластинки в области фронтального отдела нижней челюсти. А также в поиске оптимального режима сканирования для диагностики внутрикостных дефектов, таких как дегисценции и фенестрации.

\section{Материалы и метолы}

Были исследованы 20 компьютерных томограмм пациентов, которым по пародонтологическим показаниями необходима было провести пластическую пародонтологическую операцию устранений рецессий десны в области фронтального отдела нижней челюсти. Рентгенологическое исследование на аппарате 3D Accuitomo 170 (J. Morita Mfg., Corp., Kyoto, Japan) было проведено в зависимости от клинической задачи 10 пациентам, у которых не планировалось в дальнейшем ортодонтическое лечение было проведено в режиме сканирования 1 (см таблицу № 1), а также 10 пациентам, которые в дальнейшем начали ортодонтическое лечение, с использованием томографа Planmeca ProMax 3D (Planmeca OY, Helsinki, Finland) в режиме сканирования 2 (см таблицу № 1).

Данные конусно-лучевой томографии получали в формате DICOM- файлов, которые в последующем обрабатывались и визуализировались с помощью программы - просмоторщика Planmeca Romexis Viewer (рисунок № 1 и 2) 


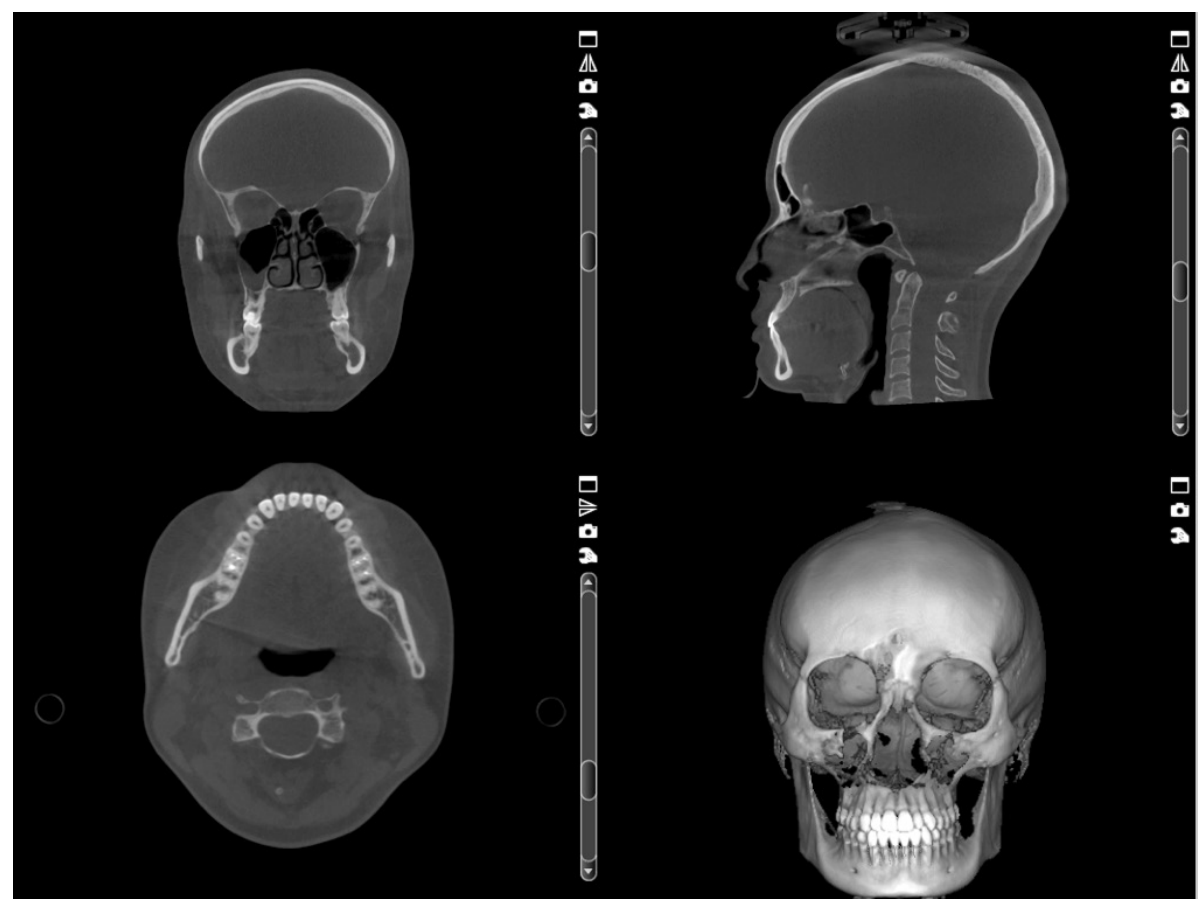

Рис. 2. Пример данных КЛКТ, полученных с помощью томографа Planmeca ProMax 3D (Planmeca OY, Helsinki, Finland)

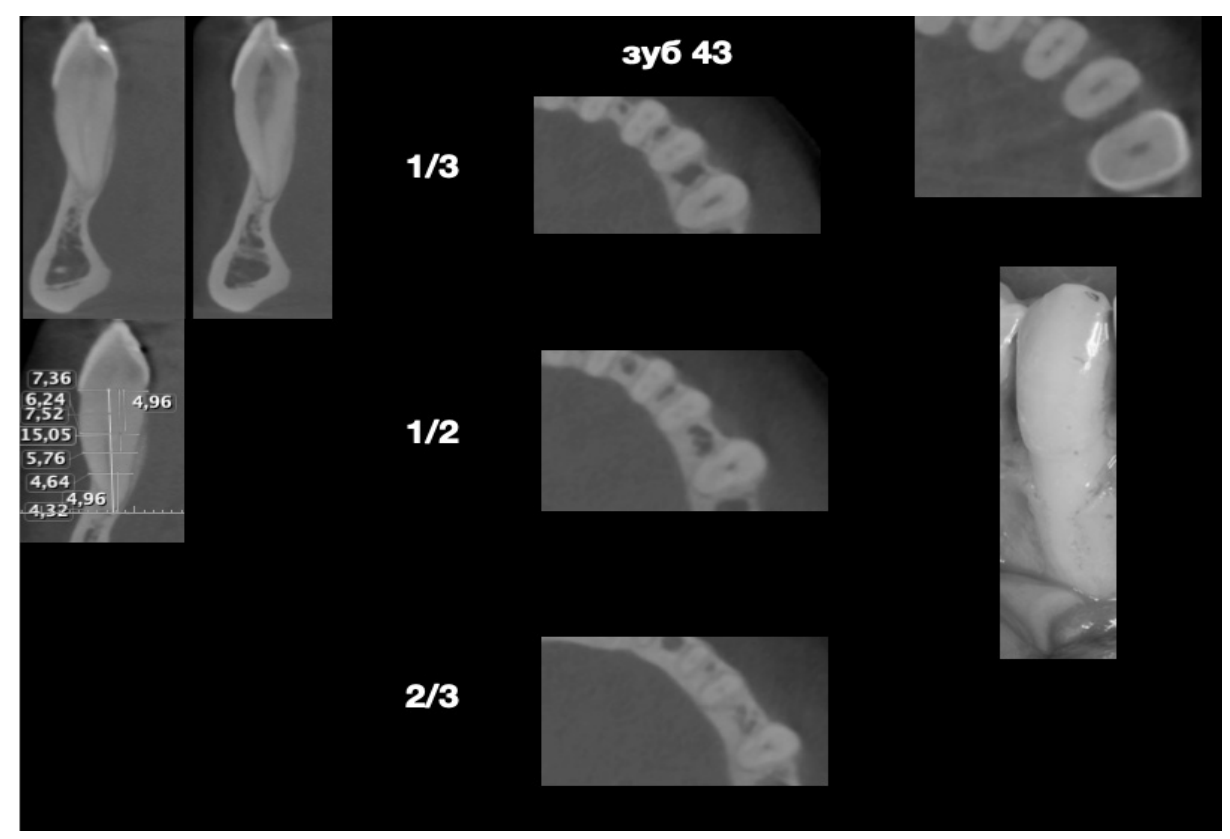

Рис. 3. Оценка морфологии наружной кортикальной пластинки

Во время хирургического вмешательства была оценена морфология наружной кортикальной пластинки в области зубов 33 и 43. Оценивали наличие, тип и высоту дефекта наружной кортикальной пластинки (рисунок № 3). Высоту дефекта измеряли с помощью пародонтального зонда с градуировкой Williams (Hu- Friedy).
Все измерения проводились на экране с увеличением 2,0 двумя независимыми исследователями. Первый исследователь - врач- стоматолог, второй исследователь - врач- рентгенолог. Воспроизводимость между значениями, измеряющими одну и ту же величину на десятых долях миллиметра (для толщины кости и расстоя- 
Таблица 2

\begin{tabular}{|l|l|l|}
\hline дефект & количество & процент \\
\hline дегисценция & 76 & 63,3 \\
\hline фенестрация & 28 & 23,3 \\
\hline Дефект отсутствует & 16 & 13,4 \\
\hline
\end{tabular}

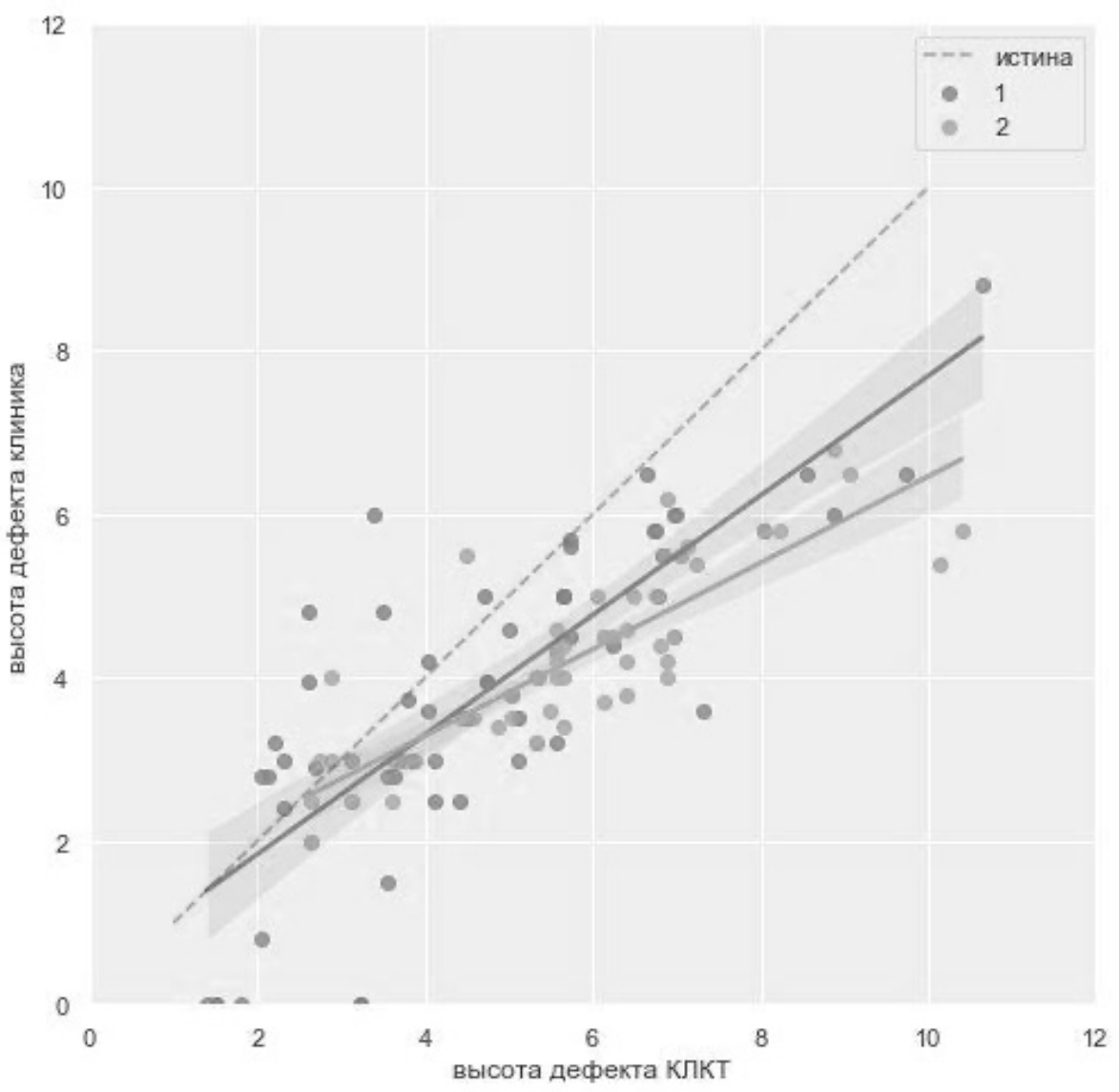

Рис. 4. Диаграмма рассеяния и линейная регрессия между истинными значениями и данными КЛКТ в разных режимах

ния) была оценена с помощью коэффициента межклассовой корреляции с доверительным интервалом 95\%. Статистически значимой разницы между экспертами не обнаружено. Полученные данные оценивали с помощью коэффициента корреляции Пирсона.

\section{Результаты и обсужмение}

Было исследовано состояние наружной кортикальной пластинки в области 120 зубов, по 60 зубов в каждой группе, в зависимости от режима сканирования.

Было обнаружено, что распространенность дигисценций во фронтальном отделе нижней челюсти выше, чем распространенность фенестраций. Данные по встречаемости дигисценций и фенестраций представлены в таблице № 2.

Распространенность дегисценций и фенестраций во фронтальном отделе нижней челюсти.

Корреляция между данными клинического осмотра и КЛКТ была выявлена с помощью коэффицента корреляции Пирсона и графически представлена на рисунке № 4.

Величина коэффициента Пирсона в группе режима сканирования с размером вокселя 0,16 составила 0,817 ( $p<0,001)$, а в группе с размером вокселя 0,4 $=0,706$ $(p<0,001)$. 
Визуализация дегисценций и фенестраций с помощью традиционных двумерных рентгенологических методов, таких как прицельная рентгенография и зонография затруднена в силу анатомических особенностей внутрикостных дефектов [3, 6, 8]. На двухмерном рентгенологическом снимке наружная кортикальная пластинка и корень зуба наслаиваются друг на друга $[9,10,13]$.

Ранее проведенными исследованиями было доказано, что конусно- лучевая компьютерная томография - оптимальный диагностический инструмент для визуализации дефектов наружной кортикальной пластинки [7, 9, 12]. Однако, исследованиями Sun et al (2015), Peterson et al (2018) было выявлена тенденция переоценки линейных размеров дефектов наружной кортикальной пластинки [10, 12], которая потвердилась и в данном исследовании. Выявлено, что при оценке данных, полученных в режиме сканирования с размером воксельного элемента $0.4 \mathrm{~mm}^{3}$, высота внутрикостных дефектов была завышена на 0,68-1,12 мм.
При оценке исследований, оценивающих точность КЛКТ важно учитывать все аспекты, включая аппараты для получения изображений КЛКТ, настройки получения, программное обеспечение, протоколы измерений и параметры визуализации, прежде чем делать выводы по нескольким исследованиям КЛКТ $[1,14]$. Данное исследование показало, что важно проявлять осторожность при использовании данных КЛКТ для интерпретации измерений дефектов наружной кортикальной пластинки нижней челюсти. Врачам-стоматологам и рентгенологам также важно найти баланс между диагностической необходимостью и лучевой нагрузкой, чтобы оптимизировать визуализацию конкретных анатомических областей. Учитывая принцип ALARA [5] необходимо провести дальнейшие исследования с большим объемом выборки для определения идеальных параметров сканирования, которые могут оптимизировать качество изображения внутрикостных дефектов без увеличения дозы лучевой нагрузки.

\section{ЛИТЕРАТУРА}

1. Величина коэффициента Пирсона в группе режима сканирования с размером вокселя 0,16 составила 0,817 ( $<<$ Ballrick JW, Palomo JM, Ruch E, Amberman BD, Hans MG. Image distortion and spatial resolution of a commercially available cone- beam computed tomography machine. Am J Orthod Dentofacial Orthop 2008;134:573-582.

2. Baumgaertel S, Palomo JM, Palomo L, Hans MG. Reliability and accuracy of cone-beam computed tomography dental measurements. Am J Orthpd Dentofacial Orthop 2009;136:19-28.

3. Braun X, Ritter L, Jervøe-Storm PM, Frentzen M. Diagnostic accuracy of CBCT for periodontal lesions. Clin Oral Investig 2014; 18: 1229-36

4. Damstra J, Fourie Z, Huddleston Slater JJ, Ren Y. Accuracy of linear measurements from cone-beam computed tomography-derived surface models of different voxel sizes. Am J Orthod Dentofacial Orthop 2010; 137: 16.e1-16

5. Farman A. ALARA still applies. Oral Surg Oral Med Oral Pathol Oral Radiol Endod 2005;100:395-397.

6. Fuhrmann R. Three-dimensional interpretation of alveolar bone dehiscences. An anatomical-radiological study — part I. J Orofac 0rthop 1996;57:62-74.

7. Kolsuz ME, Bagis N, Orhan K, Avsever H, Demiralp KÖ. Comparison of the influence of FOV sizes and different voxel resolutions for the assessment of periodontal defects. Dentomaxillofac Radiol. 2015;44(7):20150070.

8. Leung CC, Palomo L, Griffith R, Hans MG. Accuracy and reliability of cone-beam computed tomography for measuring alveolar bone height and detecting bony dehiscences and fenestrations. Am J Orthod Dentofacial Orthop 2010;137(suppl 4): s109-s119.

9. Patcas R, Mu $\bigotimes$ Iler L, Ullrich 0, Peltoma $\nabla$ ki T. Accuracy of cone-beam computed tomography at different resolutions assessed on the bony covering of the mandibular anterior teeth. Am J Orthod Dentofacial Orthop 2012; 141: 41-50.

10. Peterson AG, Wang M, Gonzalez S, Covell DA Jr, Katancik J, Sehgal HS. An In Vivo and Cone Beam Computed Tomography Investigation of the Accuracy in Measuring Alveolar Bone Height and Detecting Dehiscence and Fenestration Defects. Int J Oral Maxillofac Implants. 2018 Nov/Dec;33(6):1296-1304.

11. Spin-Neto R, Gotfredsen E, Wenzel A. Impact of voxel size variation on CBCT-based diagnostic outcome in dentistry: a sys- tematic review. J Digit Imaging 2012

12. Sun Z, Smith T, Kortam S, Kim DG, Tee BC, Fields H. Effect of bone thickness on alveolar bone-height measurements from cone- beam computed tomography images. Am J Orthod Dentofacial Orthop 2011; 139: 117-27.

13. Timock AM, CookV, McDonald T, et al. Accuracy and reliability of buccal bone height and thickness measurements from cone-beam computed tomography imaging. Am J Orthod Dentofacial Orthop 2011;140:734-744.

14. Wood R, Sun Z, Chaudhry J, et al. Factors affecting the accuracy of buccal alveolar bone height measurements from cone-beam computed tomography images. Am J Orthod Dentofacial Orthop 2013;143:353-363. 